Research

Open Access

\title{
Use of the probiotic Lactobacillus plantarum 299 to reduce pathogenic bacteria in the oropharynx of intubated patients: a randomised controlled open pilot study
}

\author{
Bengt Klarin ${ }^{1}$, Göran Molin², Bengt Jeppsson ${ }^{3}$ and Anders Larsson 4
}

\author{
${ }^{1}$ Department of Anaesthesiology and Intensive Care, University Hospital, SE-221 85 Lund, Sweden \\ ${ }^{2}$ Applied Nutrition and Food Chemistry, Lund University, Box 117, SE-221 00 Lund, Sweden \\ ${ }^{3}$ Department of Surgery, University Hospital, SE-205 02 Malmö, Sweden \\ ${ }^{4}$ Department of Anaesthesiology and Intensive Care, Aalborg Hospital, Århus University Hospitals, DK-9000 Aalborg, Denmark \\ Corresponding author: Bengt Klarin, Bengt.Klarin@med.lu.se
}

Received: 25 Jun 2008 Revisions requested: 28 Jul 2008 Revisions received: 27 Aug 2008 Accepted: 6 Nov 2008 Published: 6 Nov 2008

Critical Care 2008, 12:R136 (doi:10.1186/cc7109)

This article is online at: http://ccforum.com/content/12/6/R136

(c) 2008 Klarin et al.; licensee BioMed Central Ltd.

This is an open access article distributed under the terms of the Creative Commons Attribution License (http://creativecommons.org/licenses/by/2.0), which permits unrestricted use, distribution, and reproduction in any medium, provided the original work is properly cited.

\begin{abstract}
Introduction Ventilator-associated pneumonia (VAP) is usually caused by aspiration of pathogenic bacteria from the oropharynx. Oral decontamination with antiseptics, such as chlorhexidine $(\mathrm{CHX})$ or antibiotics, has been used as prophylaxis against this complication. We hypothesised that the probiotic bacteria Lactobacillus plantarum 299 (Lp299) would be as efficient as $\mathrm{CHX}$ in reducing the pathogenic bacterial load in the oropharynx of tracheally intubated, mechanically ventilated, critically ill patients.
\end{abstract}

Methods Fifty critically ill patients on mechanical ventilation were randomised to either oral mechanical cleansing followed by washing with $0.1 \% \mathrm{CHX}$ solution or to the same cleansing procedure followed by oral application of an emulsion of Lp299. Samples for microbiological analyses were taken from the oropharynx and trachea at inclusion and at defined intervals thereafter.

Results Potentially pathogenic bacteria that were not present at inclusion were identified in oropharyngeal samples from eight of the patients treated with Lp299 and 13 of those treated with $\mathrm{CHX}(p=0.13)$. Analysis of tracheal samples yielded similar results. Lp299 was recovered from the oropharynx of all patients in the Lp299 group.

Conclusions In this pilot study, we found no difference between the effect of Lp299 and CHX used in oral care procedures, when we examined the effects of those agents on colonisation of potentially pathogenic bacteria in the oropharynx of intubated, mechanically ventilated patients.

\section{Introduction}

Ventilator-associated pneumonia (VAP) is a common complication in intubated, mechanically ventilated patients in intensive care units (ICUs). VAP is connected to longer ICU and hospital stays, additional costs and high mortality, and the risk of developing this condition increases by $1 \%$ with each additional day of mechanical ventilation $[1,2]$.

The major cause of VAP is aspiration of either microorganisms from the oropharynx or fragments of biofilms from the endotracheal tube. Formation of such biofilms can be delayed, but not prevented, by the use of tubes with special coatings [3].
Selective decontamination using antibiotics in the oral cavity alone [4-6] or throughout the gastrointestinal (GI) tract $[7,8]$, has been shown to lower the incidence of VAP and reduce mortality. However, the use of such procedures is limited due to the risk of bacteria developing resistance to the antibiotics $[9,10]$. In recent meta-analyses, it was concluded that oral decontamination with chlorhexidine $(\mathrm{CHX})$ could prevent VAP [11], but that strategy does not reduce the time on the ventilator, the length of stay (LOS) in the ICU or rates of mortality [12]. Thus, there is a need for alternative approaches to lower the oropharyngeal load of pathogenic microorganisms as a means of decreasing the risk of VAP. 
For decades, probiotics have been given enterally to improve the microbiotic flora in the Gl tract. However, in recent years orally administered probiotics have also been shown to reduce the number of bacteria and yeast in biofilms on vocal prostheses $[13,14]$. Therefore, we hypothesised that swabbing the oral mucosa with probiotics would be an effective (and microbiologically attractive) method of reducing pathogenic oral microorganisms in tracheally intubated, mechanically ventilated, critically ill patients.

The primary aim of the present pilot study was to evaluate the feasibility and safety of an oral care procedure using the probiotic Lactobacillus plantarum 299 (Lp299) (DSM 6595) in this patient category. Like the genomically closely related strain L plantarum 299v (DSM 9843), Lp299 has been shown to adhere to the mucosa throughout the Gl tract [15-17]. Another objective of this preliminary investigation was to obtain an estimate of the number of patients needed for a definitive study examining the effectiveness of oral Lp299 in reducing the incidence of VAP.

\section{Materials and methods}

The study was approved by the Human Ethics Committee of Lund University and was performed in compliance with the Helsinki Declaration. Good clinical practice and the International Conference on Harmonisation Guidance were applied and the investigation was carried out in the ICU of the Department of Anaesthesiology and Intensive Care, University Hospital, Lund, Sweden. Informed consent was obtained from the patients or their next of kin. Further consent was not obtained from patients as they had recovered, as this was not required by the Human Ethics Committee.

The patients were randomised into groups of 10 to receive either the department's standard oral treatment (the control group) or the study treatment with Lp299 (the Lp group). The day of inclusion was designated day 1 . To be included in the study, patients had to fulfil the following criteria: 18 years of age or older; critically ill with an anticipated need for mechanical ventilation of at least 24 hours; not moribund; not suffering from pneumonia at admission; no fractures in the facial skeleton or the base of the skull; no oral ulcers; not immune deficient; not a carrier of HIV or viral hepatitis.

After screening, patients were included when ventilation and circulation had been stabilised and before the first oral care procedure. Oral care was performed twice a day. The control group was treated according to the department's standard protocol: dental prostheses were removed; secretions were removed by suction; teeth were brushed using toothpaste (Zendium, Opus Health Care, Malmö, Sweden); all mucosal surfaces were cleansed with swabs that had been moistened with a $1 \mathrm{mg} / \mathrm{ml} \mathrm{CHX}$ solution (Hexident, Ipex, Solna, Sweden). In the Lp group the initial mechanical steps were the same as in the control group, but the subsequent cleansing was instead performed with gauze swabs soaked in carbonated bottled water, after which Lp299 was applied to the mucosal surface of the oral cavity. This was performed using two gauze swabs (one for each side of the oral cavity), which had been allowed to absorb $10 \mathrm{ml}$ of a solution containing a total of $10^{10}$ colony-forming units (CFU) of Lp299. Excess suspension was not removed. In both groups, when necessary between the oral care procedures, secretions were removed by suctioning and gauze swabs moistened with carbonated bottled water were used to wipe off remaining secretions.

Cultures were taken from the oropharynx and from the trachea at inclusion. Sampling was repeated before the oral care procedures on days $2,3,5,7,10,14$ and 21 in patients that were still mechanically ventilated. If a patient was extubated on a non-culture day, cultures were taken before the extubation. One set of cultures was analysed according to normal routines at the Department of Clinical Microbiology, University Hospital. Another set was sent blinded to the research laboratory at Probi AB, Lund, Sweden for identification and quantification of total CFU of lactobacilli and identification of Lp299. Viable counts of all lactobacilli were obtained on Rogosa agar (Oxoid, Basingstoke, Hampshire, England) incubated anaerobically at $37^{\circ} \mathrm{C}$ for three days. Colonies suspected to be Lp299 (large, creamy white-yellowish and somewhat irregular in shape) were selected and identified by randomly amplified polymorphic DNA typing [18].

The patients were placed in a semi-recumbent position and were ventilated in pressure control or pressure support mode by a Servo ventilator (Maquet $A B$, Sweden) via a heat moisture exchange filter (Barrierbac "S", Mallinckrodt DAR, Mirandola, Italy). A closed suction system (TRACH-Care 72, Ballard Medical Products, Draper, UT, USA) was used. The patients inhaled $2.5 \mathrm{mg}$ salbutamol (GlaxoSmithKline, Solna, Sweden) and $0.5 \mathrm{mg}$ ipratropium (Boehringer Ingelheim, Stockholm, Sweden) every six hours.

Chest radiographs were obtained after tracheal intubation and thereafter when clinically indicated. Lung function was assessed using the Lung Injury Score (LIS) [19]. Blood gases were obtained at least three times a day and were analysed at the ICU. Samples for white blood cell (WBC) counts and Creactive protein (CRP) were collected daily and analysed at the hospital clinical chemistry laboratory.

Enteral nutrition was started and increased according to the department's protocol. The amount of enteral formula given and the total volume of other enterally administered fluids were recorded. All patients received intravenous ezomprazol (Astra Zeneca, Södertälje, Sweden) as stress ulcer prophylaxis from admission until enteral nutrition was fully established (ie, for three to four days). 
The study was neither intended nor powered for assessment of differences in the frequency of VAP. However, it was aimed at obtaining a basis for estimating the number of patients needed for a larger investigation in which VAP also constitutes a parameter. The following criteria were used to identify VAP: a new, persistent or progressive infiltrate on chest radiograph combined with at least three of the other four criteria; a purulent tracheal aspirate; positive culture of tracheal aspirates occurring after 48 hours of mechanical ventilation; rectal or urine bladder temperature higher than $38.0^{\circ} \mathrm{C}$ or less than $35.5^{\circ} \mathrm{C}$; WBC count more than 12 or less than $3[4,20]$.

\section{Statistics}

Because no previous investigation has examined the effect of probiotics in this context, we estimated that 20 patients in each group would be sufficient to assess the safety, important positive effects and possible side effects, and to give an indication of the number of patients that would be needed in a definitive study. Statistical methods were chosen after consulting a biostatistician, and the statistical analyses were performed using Statistica 6.0 (StatSoft, Tulsa, OK, USA). Student's t-test was used for the daily comparisons (days 1 to 9 ) of the parameters. Fisher's exact test was employed to compare the results of microbiological cultures. $p<0.05$ was considered significant.

\section{Results}

After screening, 50 patients were included in the study. Consent was withdrawn by two patients and another three were transferred to other ICUs shortly after inclusion. For one patient in the control group, samples were obtained only at inclusion. Altogether, 23 patients in the Lp group and 21 in the control group completed the study.

All patients were orotracheally intubated. Two in each group were reintubated, and two in the Lp group and one in the control group were tracheotomised (on days 3,16 and 3 , respectively). The proportion of patients receiving enteric nutrition and the volumes given were similar in the two groups. The patients in both groups were treated with antibiotics at the discretion of the attending physician and changes were made in compliance with culture results. Cefuroxime was the most common antibiotic used in both groups, followed by imipenem. Three patients in each group received piperacillin/tazobactam, and other antibiotics or combinations were administered to some patients in each of the two groups. Three patients did not receive any antibiotics at admission, and one of those three was not treated with antibiotics during the stay in the ICU. Ten patients in each group received corticosteroids for one or more days.

As indicated in Table 1, there were no significant differences in age or gender between the groups. Also, the admission diagnoses were similar in the two groups, as were the Acute Pathophysiology and Chronic Health Evaluation (APACHE) II scores. Some differences were found in the Sequential Organ Failure Assessment (SOFA) scores in favour of the Lp patients (data not shown). The two groups did not differ significantly with regard to the number of ventilator days, LOS, or ICU or inhospital mortality (Table 1). No deaths were caused by respiratory complications and no additional deaths occurred within six months.

No differences in WBC counts were found between the groups. Furthermore, the groups did not differ with regard to changes in CRP, although the absolute values were higher for the controls on day 3 .

No significant differences between the two groups were found when considering microbiological findings of the oropharyngeal and tracheal samples taken at inclusion. The same species were identified in samples from both the oropharynx and the trachea of six Lp patients and three controls. Subsequent oropharyngeal samples from eight $L p$ patients and from 13 controls contained enteric species that had not been present in the inclusion samples from those patients $(p=0.13)$ (Table 2). Two or three emerging species (enterococci species and enterobacteriaceae species) were found in two patients in the Lp group and seven control patients (Figure 1). Culture analysis of the tracheal samples identified emerging species in seven Lp patients and nine controls. Other comparisons of the culture results were similar. Figure 2 shows the distribution of the positive cultures according to study day and sampling site.

Lp299 was found in the oropharyngeal samples from all of the patients in the Lp group (21 of 23 patients on day 2). In addition, Lp299 was identified in the tracheal secretion samples from 13 of the patients in the Lp group (56\%), and enteric bacteria were also found in six of those subjects. Five patients in the Lp group died in the ICU, and Lp299 was identified in the tracheal samples from one of those individuals, whereas no enteric bacteria were recovered from the trachea of any of those five patients.

Considering patients in both groups, a comparison of those with positive findings and those with negative findings in cultures of tracheal secretions (results reported by the microbiology laboratory) indicated a significantly lower number of ventilator days $(p<0.001)$ in the non-colonised subjects. VAP was identified in one patient in the Lp299 group and in three patients in the $\mathrm{CHX}$ group.

\section{Discussion}

This pilot study shows that it is feasible and safe to use Lp299 as an adjunct in the oral care of intubated patients. When we compared patients subjected to an Lp299-based oral care procedure with those who underwent the standard $\mathrm{CHX}$ based oral treatment used at the department, we did not find any significant difference in the incidence of emerging, potentially pathogenic bacteria in the oropharynx or trachea. The 
Patient characteristics and admission diagnosis

\begin{tabular}{|c|c|c|}
\hline & Lp299 group & Control group \\
\hline Age & 70 (20 to 87$)$ & 70 (43 to 81$)$ \\
\hline Sex M/F & $13 / 10$ & $9 / 12$ \\
\hline APACHE II score & 22 (11 to 39$)$ & $27(9$ to 37$)$ \\
\hline ICU mortality & $5 / 23$ & $4 / 21$ \\
\hline In-hospital mortality & $5 / 23$ & $6 / 21$ \\
\hline ICU stay (days) & $7.7(1.3$ to 26.1$)$ & $6.6(1.3$ to 16.0$)$ \\
\hline Ventilator days & $5.8(1.0$ to 23.8$)$ & $4.3(1.0$ to 15.2 \\
\hline Diagnosis at admission & Lp299 group & Control group \\
\hline Sepsis, septicaemia & 6 & 5 \\
\hline Other infections & 2 & 1 \\
\hline Cardiological: arrests and insufficiencies & 5 & 4 \\
\hline Respiratory insufficiencies & 3 & 5 \\
\hline Abdominal & 1 & 2 \\
\hline Vascular (emergency aneurysms) & 0 & 3 \\
\hline Trauma & 3 & 0 \\
\hline Other & 3 & 1 \\
\hline
\end{tabular}

Data are presented as median (range) except for sex and death rates.

Differences are not significant.

APACHE = Acute Pathophysiology and Chronic Health Evaluation; ICU = intensive care units; Lp299 = Lactobacillus plantarum 299.

emerging bacteria were, as expected, mainly Gram-negative species.

The use of $\mathrm{CHX}$ in oral care procedures is considered to be an effective method to reduce pathogens in the oropharynx and to prevent VAP $[11,12]$. Aspiration of pathogenic bacteria constitutes the main cause of VAP, and thus reducing the occurrence of such microorganisms in the oropharynx should lower the rate of VAP. In our study, pathogenic enteric bacteria appeared in fewer the patients in the Lp299 group (38\%) than in the CHX group (65\%). This indicates that Lp299 might be able to lower the rate of infection with such harmful microbes and in turn lead to fewer cases of VAP. As anticipated, the difference in the incidence of VAP between the treatment groups in our study (one case in the Lp299 group and three in the $\mathrm{CHX}$ group) was inconclusive.

It should be mentioned that there are some common side effects associated with $\mathrm{CHX}$ use in oral care, including discoloration of the teeth, a burning sensation on the tongue and irritation of the mucosa [21,22]. More serious but rare adverse effects are local allergic reactions in the mouth and throat. Of particular importance is that $\mathrm{CHX}$ shows little activity against Gram-negative bacteria [23]. Moreover, it is diluted and inactivated by saliva [24], and since bacteria can be resistant to $\mathrm{CHX}$, a low concentration (which will regularly occur between oral care treatments) represents an additional risk of selection and emergence of resistant strains. What is even more alarming is that bacteria strains that are not susceptible to common antibiotics, such as methicillin-resistant Staphylococcus aureus (MRSA) also often carry genes for resistance to $\mathrm{CHX}$ [25]. $L$ plantarum strains are genetically stabile and are not likely to incorporate resistance genes or plasmids or to transfer genetic material, characteristics that are related to their inherent resistance to certain antibiotics and to other species. Consequently $L$ plantarum does not contribute to the development of antibiotic-resistant strains. In humans, lactobacilli colonise the oropharynx soon after birth, and thereafter constitute part of the normal oropharyngeal flora and, accordingly, these bacteria will enter the lower respiratory tract whenever an aspiration occurs. Other strains of lactobacillus than Lp299 have in immunocompromised patients been associated with severe infections such as endocarditis [26-28].

A limitation of our study is that we did not perform surveillance blood cultures, although the Lp299 aspirated did not produce any detectable infiltrates indicating pneumonia or bacteraemia. Furthermore, aspiration of Lp299 alone did not influence the oxygenation index, LOS or days of mechanical ventilation. Notably, the genomically closely related $L$ plantarum $299 \mathrm{v}$, has been found to be safe in an animal model of endocarditis [29]. In that study, $L$ plantarum 299 v could not be detected in the 
Table 2

Number of positive findings of bacteria species at inclusion and in subsequent samples

\begin{tabular}{|c|c|c|c|c|c|c|c|c|}
\hline \multirow{3}{*}{ Species } & \multicolumn{4}{|c|}{ Throat samples } & \multicolumn{4}{|c|}{ Tracheal secretions } \\
\hline & \multicolumn{2}{|c|}{ Inclusion } & \multicolumn{2}{|c|}{ Subsequent } & \multicolumn{2}{|c|}{ Inclusion } & \multicolumn{2}{|c|}{ Subsequent } \\
\hline & Lp & C & Lp & C & Lp & C & Lp & C \\
\hline 1 Haemophilus influenzae & 1 & 0 & 0 & 0 & 1 & 2 & 1 & 0 \\
\hline 2 Moraxella catarrhalis & 0 & 0 & 0 & 0 & 0 & 1 & 1 & 0 \\
\hline 3 Beta-Streptococcus group G & 1 & 0 & 1 & 0 & 0 & 0 & 0 & 0 \\
\hline 4 Streptococcus pneumoniae & 1 & 0 & 1 & 0 & 2 & 0 & 0 & 0 \\
\hline 5 Streptococcus pyogenes & 0 & 1 & 0 & 0 & 0 & 1 & 0 & 0 \\
\hline 1-5 Airway bacteria & 3 & 1 & 2 & 0 & 3 & 4 & 2 & 0 \\
\hline 6 Staphylococcus aureus & 6 & 2 & 1 & 0 & 3 & 0 & 2 & 0 \\
\hline 7 Citrobacter species & 0 & 0 & 1 & 0 & 0 & 0 & 1 & 0 \\
\hline 8 Escherichia coli & 1 & 2 & 1 & 2 & 1 & 1 & 0 & 1 \\
\hline 9 Enterobacter aerogenes & 1 & 2 & 0 & 0 & 0 & 0 & 0 & 1 \\
\hline 10 Enterobacter cloacae & 1 & 1 & 0 & 2 & 1 & 1 & 0 & 1 \\
\hline 11 Hafnia alvei & 0 & 0 & 0 & 1 & 0 & 0 & 0 & 0 \\
\hline 12 Klebsiella oxytoca & 0 & 0 & 1 & 0 & 0 & 1 & 0 & 1 \\
\hline 13 Morganella morgani & 0 & 0 & 0 & 1 & 0 & 0 & 0 & 0 \\
\hline 14 Proteus mirabilis & 0 & 0 & 1 & 1 & 1 & 0 & 0 & 1 \\
\hline 15 Proteus vulgaris & 0 & 0 & 1 & 0 & 0 & 0 & 1 & 0 \\
\hline 16 Pseudomonas aeruginosa & 0 & 0 & 1 & 2 & 2 & 1 & 0 & 1 \\
\hline 17 Pseudomonas species & 0 & 0 & 1 & 1 & 0 & 0 & 2 & 0 \\
\hline 18 Serratia marcescens & 0 & 0 & 0 & 0 & 0 & 0 & 1 & 0 \\
\hline 19 Serratia species & 0 & 0 & 0 & 0 & 1 & 0 & 0 & 0 \\
\hline 20 Stenotrophomonas maltophilia & 1 & 0 & 0 & 2 & 0 & 0 & 1 & 1 \\
\hline 21 Streptococcus agalactiae & 1 & 1 & 0 & 0 & 0 & 0 & 0 & 1 \\
\hline 22 Enterococcus faecalis & 0 & 0 & 3 & 3 & 0 & 0 & 1 & 2 \\
\hline 23 Enterococcus faecium & 1 & 0 & 1 & 2 & 0 & 0 & 0 & 2 \\
\hline 7-23 Enteric bacteria & 6 & 6 & 11 & 17 & 6 & 4 & 7 & 12 \\
\hline 24 Candida albicans & 5 & 4 & 5 & 9 & 3 & 7 & 5 & 5 \\
\hline 25 Candida parapsilosis & 0 & 0 & 1 & 0 & 0 & 0 & 1 & 0 \\
\hline 26 Candida tropicalis & 0 & 0 & 0 & 0 & 0 & 1 & 0 & 0 \\
\hline 24-26 Fungi & 5 & 4 & 6 & 9 & 3 & 8 & 6 & 5 \\
\hline
\end{tabular}

Only the first sample in which the species was identified is included in the presented data.

All the isolated Staphylococcus aureus strains were non-MRSA.

No significant differences were found between the two groups.

$\mathrm{Lp}=$ patients treated with Lactobacillus plantarum 299; $\mathrm{C}=$ control patients treated with chlorhexidine.

blood or heart of the laboratory animals, nor on implanted catheters 96 hours after intravenous injection of the bacteria. Both Lp299 and $L$ plantarum 299v have also been proven safe for enteral use in the ICU setting [16,30-34].
Furthermore, except for the calculated risk of aspiration, so far we have not seen any side effects of using Lp299 as an alternative in oral care. It may be more effective to add other probiotic bacteria to the treatment suspension, but at present we do 
Figure 1

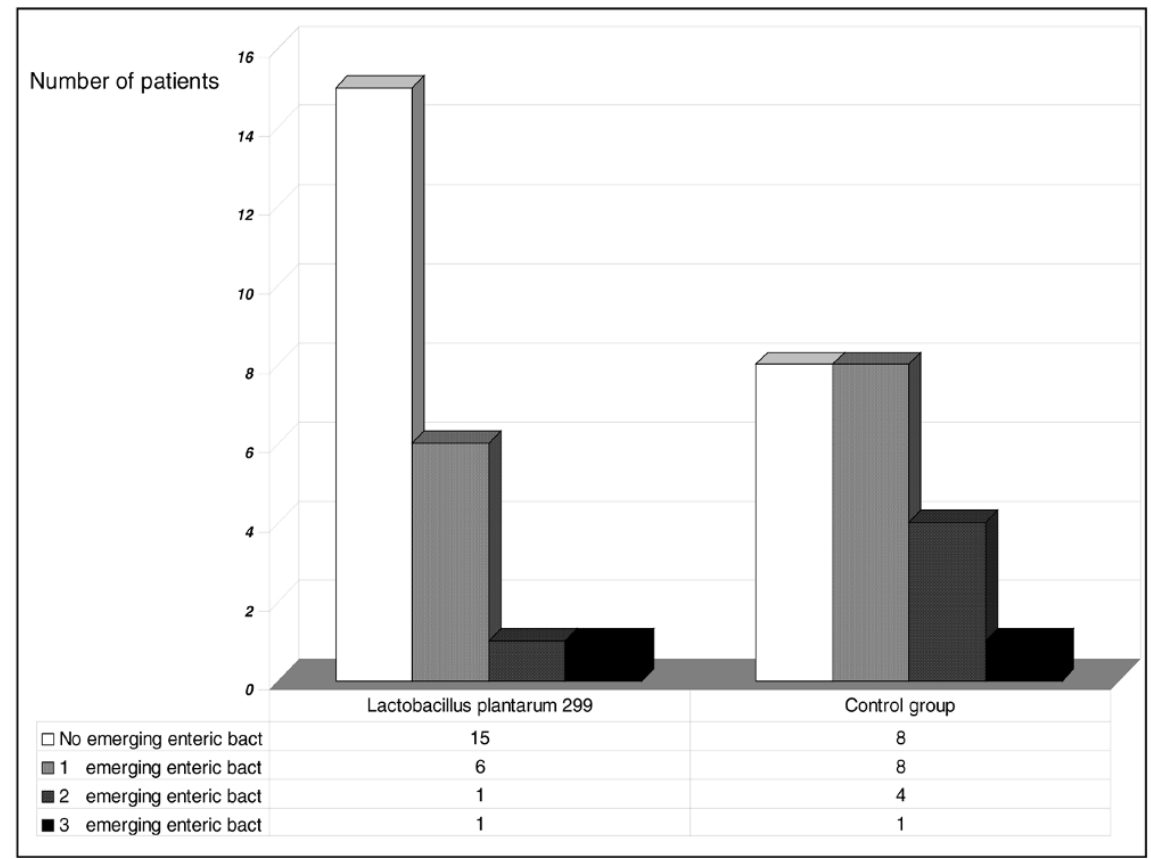

Results of oropharyngeal cultures. Number of patients with and without emerging enteric bacteria, not identified at inclusion. No new enteric species (ie, taxa not found at inclusion) appeared in 65\% (15 of 23) of the patients in the Lp299 group compared with $38 \%$ (8 of 21 ) in the control group.

not consider that approach to be safe, because it was recently found that enteral administration of a mixture of six strains of probiotics (none of them $L$ plantarum) was associated with increased mortality in patients with severe pancreatitis [35]. In

\section{Figure 2}

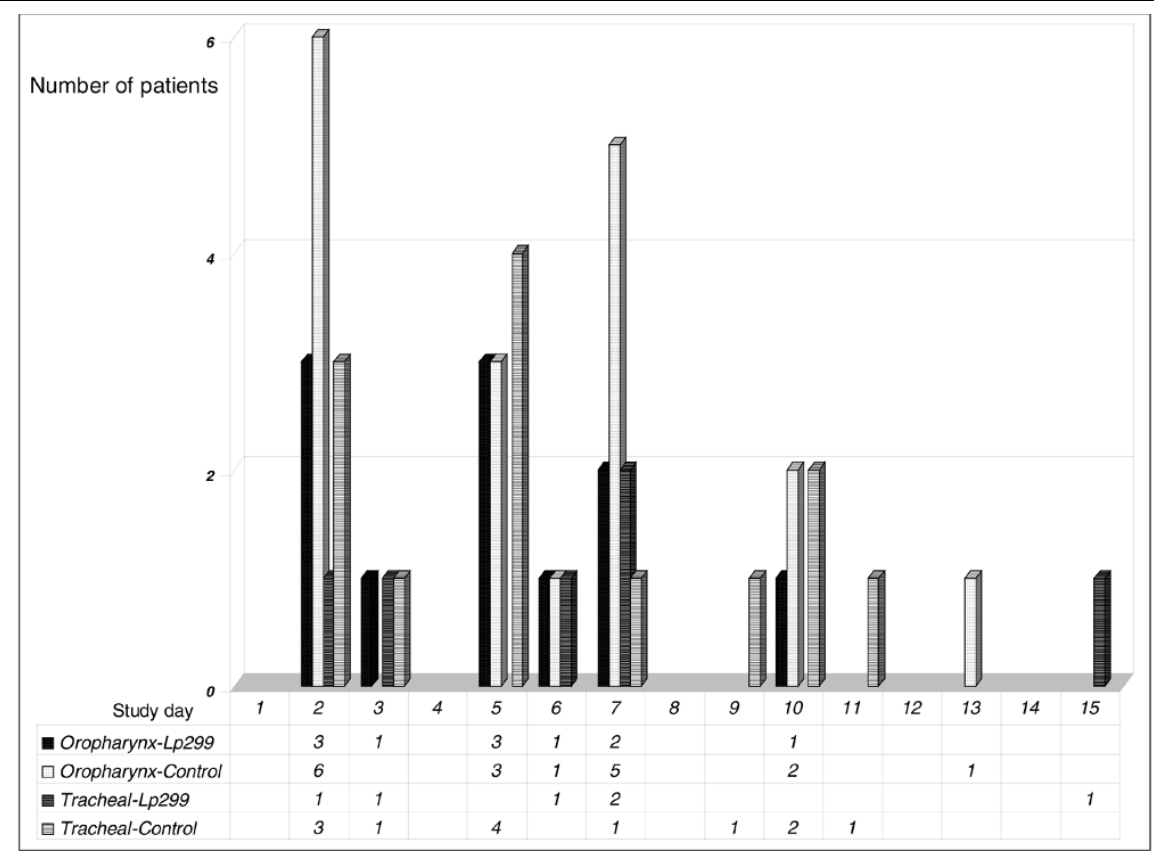

Distribution of the findings of emerging enteric bacteria. On the first days of ICU care, identified emerging enteric species were twice as many in the control patients. Despite a gradual decrease in the number of patients remaining in the study (similar in both groups), new cases of tracheal infection appeared in the latter part of the study period, primarily in the control group. 
contrast to those results, studies of $L$ plantarum 299 and $299 \mathrm{v}$ given enterally to critically ill patients have not revealed any adverse effects of those strains [16,30-34]. Also, although we did not remove excess Lp299 suspension after the oral care procedure, some of the bacteria must have reached the Gl tract, where they probably had a positive influence on the microflora. A combination of enteral and oral treatments would probably have a greater impact on the oral flora, because if any gastric content is regurgitated, it is likely to have a lower content of potentially pathogenic bacteria.

The oral care procedure in the present study was performed twice a day, which seems to correspond to the protocols in use in many ICUs [11], although it is plausible that even better results can be obtained by treating more frequently, as performed by Koeman and colleagues [36]. According to most of the relevant studies in the literature, as well as a meta-analysis $[11,12]$ different preparations and concentrations of $\mathrm{CHX}$ have been effective in reducing the incidence of VAP.

Lactobacillus species can be detected in interdental spaces, plaques and carious lesions [37], but we have found no data in the literature that seem to suggest a link between lactobacilli and initiation of caries. On the contrary, two Finnish studies have shown improved dental status and lowered counts of Streptococcus mutans in school children who consumed milk or cheese containing Lactobacillus rhamnosus GG $[38,39]$. Furthermore, in an investigation of different species of lactobacillus, it was observed that $L$ plantarum strains had the most pronounced antimicrobial effect on $S$ mutans, and they were also highly efficacious against other pathogens that are frequently found in periodontal disease [40].

The present results indicate that Lp299 might be used as a component of oral care in intubated ICU patients. Besides offering a promising alternative to antiseptics like $\mathrm{CHX}$, a probiotic that adheres to the oral mucosa will be able to counteract potentially pathogenic bacteria 24 hours a day, which is superior to the fairly short-term effect of orally applied chemical agents.

Clearly, it is also important to point out that the findings of this pilot study must be interpreted with great caution, and the trends indicated by the data must and will be further examined in a larger investigation. Nevertheless, our main objectives have been met, because we found that Lp299 did become established in the oral cavity, it had no apparent adverse effects and the results provide a basis for calculating the number of patients needed to test the trends observed in the planned definitive study.

\section{Conclusion}

Based on the results of this pilot study, we conclude that the probiotic bacterium Lp299 constitutes a feasible and safe agent for oral care. Also, it seems that Lp299 is as effective as
$\mathrm{CHX}$ in mitigating colonisation with pathogenic bacteria in the oropharynx of intubated ICU patients.

\section{Key messages \\ - Lp299 might be as effective as $\mathrm{CHX}$ in reducing the incidence of emerging potentially pathogenic bacteria in the oropharynx of intubated, mechanically ventilated, critically ill patients. \\ - We did not observe any adverse effects of the oral care procedure involving use of the probiotic bacterium Lp299.}

\section{Competing interests}

Probi $A B$ provided the study product as an unconditional grant and performed bacterial analyses. Probi $A B$ has also done the same in earlier studies performed by BK. BJ and GM are shareholders in Probi $A B$, and $G M$ resigned as a board member in 2005. Probi AB holds the patent for the investigated bacterium, but there is no patent regarding the studied application. Other financially related matters regarding GM's position as Professor at Lund University is regulated in a central and official agreement between Lund University and Probi AB.

\section{Authors' contributions}

BK was the prime investigator and did most of the planning and performance of the study. BK handled the primary data and did most of the statistical work, and also collaborated with $\mathrm{GM}, \mathrm{BJ}$ and $\mathrm{AL}$ to prepare and finalise the manuscript. GM contributed substantially to the analysis of the results of the bacterial cultures and completion of the manuscript. BJ took part in planning of the study and in finalising the manuscript. AL helped plan the study and was very active in preparing and competing the manuscript.

\section{Acknowledgements}

We are very grateful to our research nurse, Anne Adolfsson, for her impressive commitment to the study. We also thank the biostatisticians at Region Skåne Competence Centre for Clinical Research for all their valuable help. This study was supported by grants from the following sources: Region Skåne, Sweden; the Scandinavian Society for Antimicrobial Chemotherapy Foundation; Probi AB, Lund, Sweden (unconditional). The trial registration is Current Controlled Trials ISRCTN00472141.

\section{References}

1. Chastre J, Fagon JY: Ventilator-associated pneumonia. $A m \mathrm{~J}$ Respir Crit Care Med 2002, 165:867-903.

2. Safdar N, Dezfulian C, Collard HR, Saint S: Clinical and economic consequences of ventilator-associated pneumonia: $A$ systematic review. Crit Care Med 2005, 33:2184-2193.

3. Rello J, Kollef M, Diaz E, Sandiumenge A, del Castillo Y, Corbella $X$, Zachskorn R: Reduced burden of bacterial airway colonization with a novel silver-coated endotracheal tube in a randomized multiple-center feasibility study. Crit Care Med 2006, 34:2766-2772.

4. Bergmans DCJJ, Bonten MJM, Gaillard CA, Paling JC, Geest S van der, van Tiel FH, Beysens AJ, de Leeuw PW, Stobberingh EE: Prevention of ventilator-associated pneumonia by oral decontamination: a prospective, randomized, double-blind, placebo- 
controlled study. Am J Respir Crit Care Med 2001, 164:382-388.

5. Abele-Horn M, Dauber A, Bauernfeind A, Russwurm W, SeyfarthMetzger L, Gleich P, Ruckdeschel G: Decrease in nosocomial pneumonia in ventilated patients by selective oropharyngeal decontamination. Intensive Care Med 1996, 23:187-195.

6. Pugin J, Auckenthaler R, Lew DP, Suter PM: Oropharyngeal decontamination decreases incidence of ventilator-associated pneumonia: A randomized, placebo-controlled, double-blind clinical trial. JAMA 1991, 265:2704-2710.

7. D'Amico R, Pifferi S, Leonetti C, Torri V, Tinazzi A, Liberati A: Effectiveness of antibiotic prophylaxis in critically ill adult patients: Systematic review of randomised controlled trials. BMJ 1998 , 316:1275-1285

8. Nathens $A B$, Marshall JC: Selective decontamination of the digestive tract in surgical patients: A systematic review of the evidence. Arch Surg 1999, 134:170-176.

9. de Jonge E, Schultz MJ, Spanjaard L, Bossuyt PMM, Vroom MB, Dankert J, Kesecioglu J: Effects of selective decontamination of digestive tract on mortality and acquisition of resistant bacteria in intensive care: A randomized controlled trial. Lancet $2003,362: 1011-1016$.

10. Kollef $\mathrm{MH}$ : Selective digestive decontamination should not be routinely employed. Chest $2003,123: 464 \mathrm{~s}-468 \mathrm{~s}$.

11. Chlebick MP, Safdar N: Topical chlorhexidine for prevention of ventilator-associated pneumonia: A meta-analysis. Crit Care Med 2007, 35:595-602.

12. Chan $E Y$, Ruest $A, O$ Meade $M$, Cook DJ: Oral decontamination for prevention of systematic review and meta-analysis pneumonia in mechanically ventilated adults. BMJ 2007, 334:889.

13. Free RH, Mei HC Van der, Dijk F, Van Weissenbruch R, Busscher $\mathrm{HJ}$, Albers FWJ: Biofilm formation on voice prostheses: In vitro influence of probiotics. Ann Otol Rhinol Laryngol 2001, 110:946-951

14. Schwandt $L Q$, van Weissenbruch $R$, Mei $H C$ van der, Busscher $\mathrm{HJ}$, Albers FWJ: Effect of dairy products on the lifetime of Provox2 voice prostheses in vitro and in vivo. Head Neck 2005, 27:471-477.

15. Johansson M-L, Molin G, Jeppsson B, Nobaek S, Ahrné S, Bengmark S: Administration of different Lactobacillus strains in fermented oatmeal soup: in vivo colonization of human intestinal mucosa and effect on the indigenous flora. Appl Environ Microbiol 1993, 59:15-20.

16. Klarin B, Johansson M-L, Molin G, Larsson A, Jeppsson B: Adhesion of the probiotic bacterium Lactobacillus plantarum 299v onto the gut mucosa in critically ill patients: a randomised open trial. Critical Care 2005, 9:R285-R293.

17. Stjernquist-Desatnik $A$, Warfving $H$, Johansson M-L: Persistence of Lactobacillus plantarum DSM 9843 on human tonsillar surface after oral administration in fermented oatmeal gruel. A pilot study. Acta Otolaryngo/ 2000, 543:215-219.

18. Johansson M-L, Quednau M, Molin G, Ahrné S: Randomly Amplified Polymorphic DNA (RAPD) for rapid typing of Lactobacillus plantarum strains. Lett Appl Microbiol 1995, 21:155-159.

19. Murray JR, Matthay MA, Luce JM, Flick R: An expanded definition of the adult respiratory distress syndrome. Am Rev Respir Dis 1988, 138:720-723.

20. Klompas M: Does This Patient Have Ventilator-Associated Pneumonia? JAMA 2007, 297:1583-1593.

21. Cankaya H, Ozen S, Kiro@lu F, Yurttas V: Effects of topical chlorhexidine applied to the rabbit nasal mucosa. Auris Nasus Larynx 2003, 30:65-69.

22. Tantipong $\mathrm{H}$, Morkchareonpong $\mathrm{C}$, Jaiyindee $\mathrm{S}$, Thamlikitkul $\mathrm{V}$ : Randomized Controlled Trial and Meta-analysis of Oral Decontamination with $2 \%$ Chlorhexidine Solution for the Prevention of Ventilator-Associated Pneumonia. Infect Control Hosp Epidemiol 2008, 29:131-136.

23. Spiikervet FKL, van Saene HKF, Panders AK, Vermey A, van Saene JJM, Mehta DM, Fidler V: Effect of chlorhexidine rinsing on the oropharyngeal ecology in patients with head and neck cancer who have irradiation mucositis. Oral Surg Oral Med Oral Pathol 1989, 67:154-161.

24. Spijkervet FK, van Saene JJ, van Saene HK, Panders AK, Vermey A, Fidler V: Chlorhexidine inactivation by saliva. Oral Surg Oral Med Oral Pathol 1990, 69:444-449.

25. Vali L, Davies SE, Lai LLG, Dave J, Amyes SGB: Frequency of biocide resistance genes, antibiotic resistance and the effect of chlorhexidine exposure on clinical methicillin-resistant Staphylococcus aureus isolates. J Antimicrob Chemother 2008 61:524-532.

26. Salvana EMT, Frank M: Lactobacillus endocarditis: Case report and review of cases reported since 1992. J Infect 2006, 53:e5-e10.

27. Salminen MK, Rautelin H, Tynkkynen S, Poussa, Saxelin M, Valtonen V: Lactobacillus bacteremia, clinical significance, and patient outcome, with special focus on probiotic $L$. rhamnosus GG. Clin Infect Dis 2004, 38:62-69.

28. Cannon JP, Lee TA, Bolanos JT, Danziger : Pathogenic relevance of Lactobacillus: a retrospective review of over 200 cases. Eur $J$ Clin Microbiol Infect Dis 2005, 24:31-40.

29. Adawi D, Molin G, Ahrne S, Jeppsson B: Safety of the Probiotic Strain Lactobacillus plantarum DSM 9843 (= strain 299v) in an Endocarditis Animal Model. Microbial Ecol Health Disease 2002, 14:50-53.

30. Rayes N, Seehofer D, Hansen S, Boucsein K, Muller AR, Serke S, Bengmark S, Neuhaus P: Early enteral supply of Lactobacillus and fiber versus selective bowel decontamination: a controlled trial in liver transplant recipients. Transplantation 2002 74:123-128

31. Oláh A, Belágyi T, Issekutz Á, Gamal ME, Bengmark S: Randomized clinical trial of specific lactobacillus and fibre supplement to early enteral nutrition in patients with acute pancreatitis. Br J Surg 2002, 89:1103-1107.

32. McNaught CE, Woodcock NP, MacFie J, Mitchell CJ: A prospective randomised study of the probiotic Lactobacillus plantarum $299 \mathrm{~V}$ on indices of gut barrier function in elective surgical patients. Gut 2002, 51:827-831.

33. McNaught CE, Woodcock NP, Anderson ADG, MacFie J: A prospective randomised trial of probiotics in critically ill patients. Clin Nutr 2005, 24:211-219.

34. Klarin B, Wullt M, Palmquist I, Molin G, Larsson A, Jeppsson B: Lactobacillus plantarum $299 \mathrm{v}$ reduces colonisation of Clostridium difficile in critically ill patients treated with antibiotics. Acta Anaesthesiol Scand 2008, 52:1096-1102.

35. Besselink MG, van Santvoort HC, Buskens E, Boermeester MA, van Goor H, Timmerman HM, Nieuwenhuijs VB, Bollen TL, van Ramshorst B, Witteman BJ, Rosman C, Ploeg RJ, Brink MA Schaapherder AF, Dejong $\mathrm{CH}$, Wahab PJ, van Laarhoven CJ, Harst E van der, van Eijck $\mathrm{CH}$, Cuesta MA, Akkermans LM, Gooszen HG for the Dutch Acute Pancreatitis Study Group: Probiotic prophylaxis in predicted severe acute pancreatitis: a randomised, double-blind, placebo-controlled trial. Lancet 2008, 371:651-659.

36. Koeman M, Ven AJAM van der, Hak E, Joore HCA, Kaasjager K, de Smet AGA, Ramsay G, Dormans TPJ, Aarts LPHJ, de Bel EE, Hustinx WNM, Tweel I van der, Hoepelman AM, Bonten MJM: Oral Decontamination with Chlorhexidine Reduces the Incidence of Ventilator-associated Pneumonia. Am J Respir Crit Care Med 2006, 173:1348-1355

37. Crossner CG, Claesson R, Johansson T: Presence of mutans streptococci and various types of lactobacilli in interdental spaces related to development of proximal carious lesions. Scand J Dent Res 1989, 97:307-315.

38. Näse L, Hatakka K, Savilahti E, Saxelin M, Pönkä A, Poussa T: Effect of long-term consumption of a probiotic bacterium Lactobacillus rhamnosus GG in milk on dental caries and caries risk in children. Caries Res 2001, 35:412-420.

39. Ahola AJ, Yli-Knuuttila H, Suomalainen T, Poussa T, Ahlström A, Meurman JH, Korpela R: Short-term consumption of probioticcontaining cheese and its effect on dental caries risk factors. Arch Oral Biol 2002, 47:799-804.

40. Köll-Klais $\mathrm{P}$, Mändar $\mathrm{R}$, Leibur $\mathrm{E}$, Marcotte $\mathrm{H}$, Hammarström L, Mikelsaar M: Oral lactobacilli in chronic periodontitis and periodontal health: species composition and antimicrobial activity. Oral Microbiol Immunol 2005, 20:354-361. 